\title{
Synthese, Röntgenstrukturanalyse und katalytische Aktivität neuer Erdalkalimetall-Alkinkomplexe
}

\begin{tabular}{|r|l|}
\hline Journal: & Zeitschrift für Anorganische und Allgemeine Chemie \\
\hline Manuscript ID: & zaac.200900159 \\
\hline Wiley - Manuscript type: & Article \\
\hline Date Submitted by the \\
Author: & 15-Mar-2009 \\
\hline Complete List of Authors: & $\begin{array}{l}\text { Schumann, Herbert; TU Berlin, Chemistry } \\
\text { Steffens, Alexandra } \\
\text { Hummert, Markus }\end{array}$ \\
\hline Keywords: & $\begin{array}{l}\text { Alkyne complexes, Calcium, Magnesium, X-ray structures, } \\
\text { Polymerization catalysts }\end{array}$ \\
\hline \multicolumn{2}{|l}{} \\
\hline
\end{tabular}

\section{今 scholaroNE" \\ Manuscript Central}


Synthese, Röntgenstrukturanalyse und katalytische Aktivität

\title{
neuer Erdalkalimetall-Alkinkomplexe
}

Synthesis, X-Ray Structures and Catalytic Activity of new Alkyne

Complexes of the Alkaline Earth Metals

\section{Herbert Schumann*, Alexandra Steffens und Markus Hummert}

Berlin, Institut für Chemie der Technischen Universität

Bei der Redaktion eingegangen am ...̈̈rz 2009

Prof. Gerd Meyer zum 60. Geburtstag gewidmet

\begin{abstract}
Mg}\left[\mathrm{N}\left(\mathrm{SiMe}_{3}\right)_{2}\right]_{2}(\mathrm{THF})_{2}$ (1) reacts with equal amounts of $\mathrm{HC} \equiv \mathrm{CPh}$ and $\mathrm{HC} \equiv \mathrm{CSiMe}_{3}$ in $\mathrm{THF}$ yielding $\left[\left(\mathrm{Me}_{3} \mathrm{Si}\right)_{2} \mathrm{NMgC} \equiv \mathrm{CPh}(\mathrm{THF})\right]_{2}$ (2) and $\left[\left(\mathrm{Me}_{3} \mathrm{Si}\right)_{2} \mathrm{NMgC} \equiv \mathrm{CSiMe}_{3}(\mathrm{THF})\right]_{2}$ (3), respectively. The reactions of $\left(\mathrm{C}_{5} \mathrm{HMe}_{4}\right) \mathrm{MgBu}(4)$, prepared in situ from $\mathrm{Mg}\left(\mathrm{C}_{5} \mathrm{HMe}_{4}\right)_{2}$ and $\mathrm{MgBu}_{2}$, with $\mathrm{HC} \equiv \mathrm{CPh}$ and $\mathrm{HC} \equiv \mathrm{CSiMe}_{3}$ in THF afford $\left[\left(\mathrm{C}_{5} \mathrm{HMe}_{4}\right) \mathrm{MgC} \equiv \mathrm{CPh}(\mathrm{THF})\right]_{2}(\mathbf{5})$ and $\left(\mathrm{C}_{5} \mathrm{HMe}_{4}\right) \mathrm{MgC} \equiv \mathrm{CSiMe}_{3}(\mathrm{THF})_{2}(\mathbf{6})$. The reaction of equivalent amounts
\end{abstract}

\footnotetext{
${ }^{*}$ Prof. Dr. H. Schumann

Institut für Chemie,

Sekr. C2, der Technischen Universität

Straße des 17. Juni 135

D-10623 Berlin

Fax: +49-(030)-31422168

E-mail: schumann@chem.tu-berlin.de
} 
of metallic $\mathrm{Ca}, \mathrm{C}_{5} \mathrm{H}_{2} \mathrm{Me}_{4}$ and $\mathrm{HC} \equiv \mathrm{CPh}$ results in the formation of $\left[\left(\mathrm{C}_{5} \mathrm{HMe}_{4}\right) \mathrm{CaC} \equiv \mathrm{CPh}(\mathrm{THF})\right]_{2}(7)$. The ${ }^{1} \mathrm{H}$ and ${ }^{13} \mathrm{C}$ NMR spectra of the new compounds as well as the single crystal X-ray structures of $\mathbf{3}, \mathbf{5}$, and $\mathbf{6}$ are reported and discussed. The alkyne complexes $3,5,6$, and 7 catalyze the polymerization of methylmethacrylate.

Key words: Alkyne complexes, Calcium, Magnesium, NMR spectroscopy, X-ray structures, Polymerization catalysts

\section{Einleitung}

Zur Synthese von für medizinische und pharmazeutische Zwecke geeigneten Polymeren benötigt man biologisch unbedenkliche Katalysatoren, da es in der Regel nicht gelingt, dieselben oder deren Abbauprodukte nach erfolgter Polymerisation restlos vom Polymer $\mathrm{zu}$ trennen [1]. Katalytisch aktive Verbindungen der am tierischen und pflanzlichen Stoffwechsel beteiligten Erdalkalimetalle Magnesium und Calcium erscheinen deshalb besonders gut geeignet. Erste Untersuchungen auf diesem Gebiet wurden in den Jahren zwischen 1970 und 1990 durchgeführt. Sie beschreiben die Polymerisation von Methylmethacrylat und Styrol mit Hilfe von fein verteiltem Calcium, Strontium und Barium als Katalysatoren [2] oder die durch $\mathrm{MgO}$ und $\mathrm{CaO}$ katalysierte Polymerisation von L-Laktid [3]. In den folgenden Jahren wurden Studien zur Polymerisation von Methylmethacrylat unter Verwendung von Dicyclopentadienylcalcium [4, 5], Bis(pentamethylcyclopentadienyl)calcium [5] oder Triphenylmethylcalcium, -strontium und -barium als Katalysatoren [6], zur Polymerisation von L-Laktid und $\varepsilon$-Caprolakton mit $\mathrm{Ca}(\mathrm{O}-i-\mathrm{Pr})_{2}$ [7], $\mathrm{Ca}(\mathrm{OMe})_{2}$ [8] oder $\mathrm{Sr}(\mathrm{O}-i-\mathrm{Pr})_{2}$ als Katalysatoren [9] sowie zur 
Copolymerisation von Polyglycolid und Polylaktid unter Verwendung katalytischer Mengen Calciumacetylacetonat [10] durchgeführt. Zuletzt zeigten die zwischen 2001 und 2003 erschienenen Arbeiten von Harder et al,, dass Benzylcalciumverbindungen die stereoselektive Polymerisation von Styrol initiieren [11].

Im Rahmen unserer Arbeiten über unterschiedlich substituierte Dicyclopentadienylverbindungen der Erdalkalimetalle [12] konnten wir feststellen, dass $\mathrm{Mg}\left(\mathrm{C}_{5} \mathrm{HMe}_{4}\right)_{2}, \mathrm{Ca}\left(\mathrm{C}_{5} \mathrm{HMe}_{4}\right)_{2}(\mathrm{THF})$ und $\mathrm{Sr}\left(\mathrm{C}_{5} \mathrm{HMe}_{4}\right)_{2}(\mathrm{THF})_{2}$ sowohl mit als auch ohne Aluminiumorganyle als Cokatalysatoren bereits bei $-50{ }^{\circ} \mathrm{C}$ Methylmethacrylat quantitativ polymerisieren [13, 14]. Für den Mechanismus der durch $\mathrm{Ca}\left(\mathrm{C}_{5} \mathrm{HMe}_{4}\right)_{2}(\mathrm{THF})$ im Beisein von $\mathrm{AlEt}_{3}$ als Cokatalysator ausgelösten lebenden Polymerisation von Methylmethacrylat schlugen wir einen Primärangriff von $\mathrm{AlEt}_{3}$ auf den Calciumkomplex unter Bildung der elektronisch ungesättigten Spezies $\left[\left(\mathrm{C}_{5} \mathrm{HMe}_{4}\right) \mathrm{CaEt}\right]$ vor, dessen Koordination an das Monomer dann den Polymerisationsprozess auslöst $[13,14]$ (Schema 1). Dieser Mechanismus legt die Vermutung nahe, dass Halbsandwichkomplexe der Erdalkalimetalle die effektiveren Katalysatoren für die Polymerisation polarer Monomere darstellen. Die bisher bekannten Komplexe dieser Art wie $\left(\mathrm{C}_{5} \mathrm{Me}_{5}\right) \mathrm{CaI}(\mathrm{THF})_{2}[15],\left[\mathrm{C}_{5} \mathrm{H}_{2}\left(\mathrm{SiMe}_{3}\right)_{3}\right] \mathrm{CaI}(\mathrm{THF})$ [16], $\left[\mathrm{C}_{5} \mathrm{H}(i-\mathrm{Pr})_{4}\right] \mathrm{CaI}(\mathrm{THF})[17],\left[\mathrm{C}_{5} \mathrm{H}(i-\mathrm{Pr})_{4}\right] \mathrm{CaC} \equiv \mathrm{CR}(\mathrm{THF})_{\mathrm{n}}\left(\mathrm{R}=\mathrm{Ph}, \mathrm{SiMe}_{3}, \mathrm{Si}(i-\mathrm{Pr})_{3}\right.$, $\left.\mathrm{SiPh}_{3}\right)$ [18] und $\left(\mathrm{C}_{5} \mathrm{H}_{5}\right) \mathrm{MgC} \equiv \mathrm{CPh}[19]$ wurden jedoch hinsichtlich ihrer katalytischen Fähigkeiten bis heute nicht getestet.

Im folgenden berichten wir über die Synthese und Charakterisierung sowie die katalytischen Eigenschaften einiger neuer Bis(trimethylsilyl)amidomagnesiumalkinsowie Tetramethylcyclopentadienylmagnesium- und -calciumalkin Derivate. 


\begin{tabular}{|l|r|r|}
\hline $\begin{array}{l}\text { \$ASQ5833185_File000000_8559 } \\
\text { 7638.doc }\end{array}$ & $-4-$ & $15.03 .200910: 32: 00$ \\
\hline
\end{tabular}

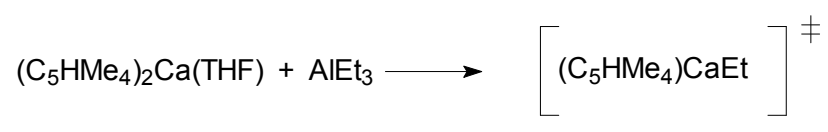
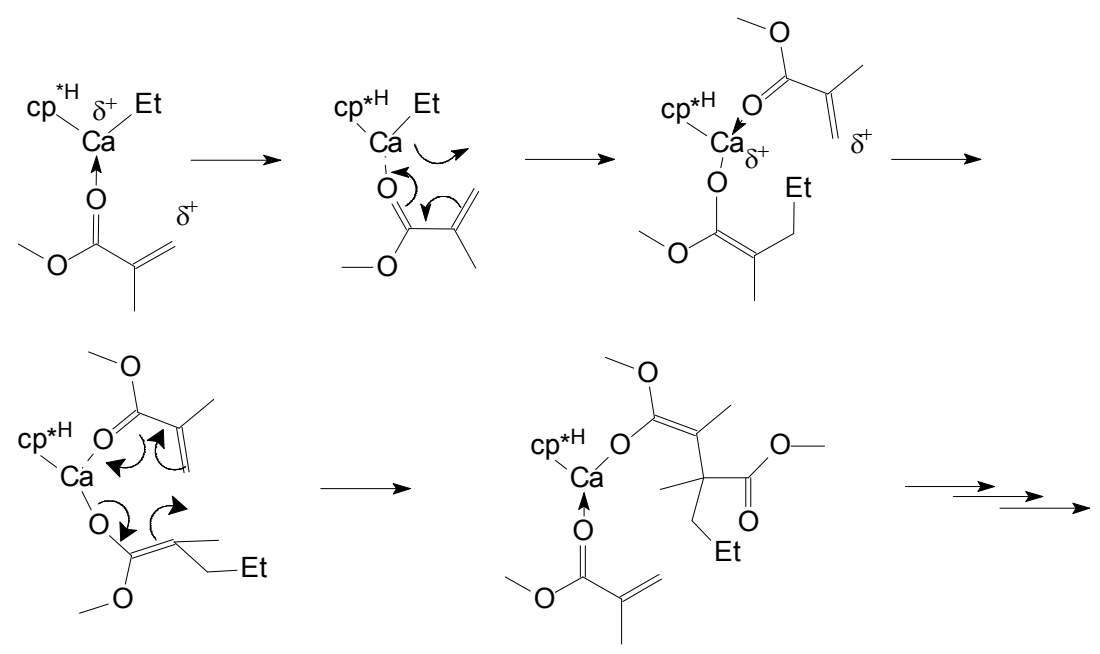

\section{Schema 1}

\section{Synthese und Eigenschaften}

Die Umsetzung equimolarer Mengen von $\mathrm{Mg}\left[\mathrm{N}\left(\mathrm{SiMe}_{3}\right)_{2}\right]_{2}(\mathrm{THF})_{2}$ (1) mit Phenylacetylen oder Trimethylsilylacetylen in Tetrahydrofuran führt unter Substitution eines Bis(trimethylsilyl)amid-Liganden durch einen Alkin-Liganden zur Bildung von $\left[\left(\mathrm{Me}_{3} \mathrm{Si}\right)_{2} \mathrm{NMgC} \equiv \mathrm{CPh}(\mathrm{THF})\right]_{2}$ (2) bzw. $\left[\left(\mathrm{Me}_{3} \mathrm{Si}\right)_{2} \mathrm{NMgC} \equiv \mathrm{CSiMe}_{3}(\mathrm{THF})\right]_{2}$ (3) allerdings in sehr unterschiedlichen Ausbeuten von 86 (2) bzw. 36\% (3) (Schema 2).

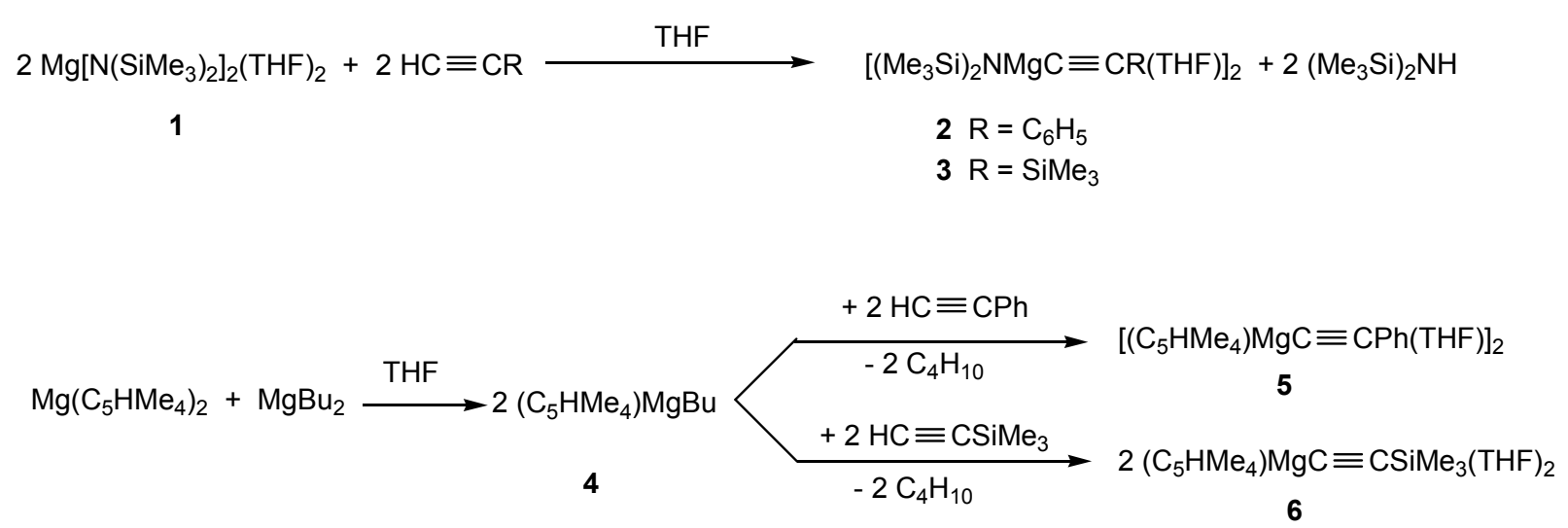

\section{Schema 2}


Die farblosen Dimeren 2 und 3 lösen sich gut in Hexan, Toluol und Tetrahydrofuran. Ihre ${ }^{1}$ H-NMR-Spektren zeigen die für die Protonen der Trimethylsilyl- und Phenylgruppen sowie des THF Liganden charakteristischen Signale in den erwarteten Bereichen. Im ${ }^{13} \mathrm{C}-\mathrm{NMR}$ Spektrum von $\mathbf{3}$ erscheinen die Signale für die beiden Acetylenkohlenstoffatome bei 134.14 und 143.79 ppm. Zur EinkristallRöntgenstrukturbestimmung geeignete Kristalle konnten nur von $\mathbf{3}$ aus Hexan erhalten werden.

Überraschenderweise ist es nicht möglich, analog zur Synthese von $\mathbf{2}$ und $\mathbf{3}$ aus $\mathrm{Mg}\left(\mathrm{C}_{5} \mathrm{HMe}_{4}\right)_{2}$ und Phenylacetylen bzw. Trimethylsilylacetylen entsprechende Tetramethylcyclopentadienylmagnesiumverbindungen zu erhalten. Dies gelingt jedoch auf dem Umweg über Butyl(tetramethylcyclopentadienyl)magnesium, $\left(\mathrm{C}_{5} \mathrm{HMe}_{4}\right) \mathrm{MgC}_{4} \mathrm{H}_{9}$ (4). Das aus equimolaren Anteilen von Dibutylmagnesium und Bis(tetramethylcyclopentadienyl)magnesium entstehende $\mathbf{4}$ reagiert in situ mit der molaren Menge von Phenylacetylen oder Trimethylsilylacetylen unter Bildung von dimerem $\left[\left(\mathrm{C}_{5} \mathrm{HMe}_{4}\right) \mathrm{MgC} \equiv \mathrm{CPh}(\mathrm{THF})\right]_{2}$ und monomerem $\left(\mathrm{C}_{5} \mathrm{HMe}_{4}\right) \mathrm{MgC} \equiv \mathrm{CSiMe}_{3}(\mathrm{THF})_{2}$ (6) (Schema 2). Die von beiden Komplexen durch Umkristallisieren aus Toluol gewonnenen farblosen, luftempfindlichen Kristalle zersetzen sich oberhalb $179{ }^{\circ} \mathrm{C}(5)$ bzw. $320{ }^{\circ} \mathrm{C}(\mathbf{6})$. Ihre ${ }^{1} \mathrm{H}$ und ${ }^{13} \mathrm{C}-\mathrm{NMR}$ Spektren bestätigen ihre Konstitution.

Der Komplex $\left[\left(\mathrm{C}_{5} \mathrm{HMe}_{4}\right) \mathrm{CaC} \equiv \mathrm{CPh}(\mathrm{THF})\right]_{2}$ (7) kann durch Einleiten von Ammoniak in ein auf $-40{ }^{\circ} \mathrm{C}$ gekühltes Reaktionsgemisch bestehend aus equimolaren Mengen Tetramethylcyclopentadien, Phenylacetylen und klein zerteiltem Calcium in THF erhalten werden (Schema 3). Die nach der Auflösung des Calciums vorliegende rosa Suspension färbt sich während des Erwärmens des Reaktionsgemisches auf 
$-6-$

15.03.2009 10:32:00

Raumtemperatur weiß. Aus der nach Filtration und Pentanwäsche zurückbehaltenen Festsubstanz lässt sich das als Nebenprodukt gebildete $\left(\mathrm{C}_{5} \mathrm{HMe}_{4}\right)_{2} \mathrm{Ca}(\mathrm{THF})_{2}$ durch Extraktion mit Toluol entfernen. Zurück bleibt reines, äußerst luftempfindliches 7 in einer Ausbeute von 38\%. Da der Komplex in Pentan, Hexan, heißem Toluol und THF weitgehend unlöslich ist, schlugen alle Versuche fehl, durch Umkristallisation Kristalle zu erhalten, die für die Röntgenstrukturanalyse geeignet gewesen wären. Das ${ }^{1} \mathrm{H}-\mathrm{NMR}$ Spektrum von 7, aufgenommen in $\mathrm{C}_{5} \mathrm{D}_{5} \mathrm{~N}$, belegt dessen angegebene Zusammensetzung.

$$
\left.\mathrm{Ca}+\mathrm{C}_{5} \mathrm{H}_{2} \mathrm{Me}_{4}+\mathrm{HC} \equiv \mathrm{CPh} \stackrel{\mathrm{fl} . \mathrm{NH}_{3}}{\longrightarrow} \underset{7}{\left[\left(\mathrm{C}_{5} \mathrm{HMe}_{4}\right) \mathrm{CaC}\right.} \underset{7}{\equiv} \mathrm{CPh}(\mathrm{THF})\right]_{2}+\mathrm{H}_{2}
$$

\section{Schema 3}

\section{Röntgenstrukturanalysen}

Die für die Röntgenstrukturanalysen von 3, 5 und $\mathbf{6}$ geeigneten Einkristalle wurden aus Hexan (3) bzw. Toluol (5, 6) erhalten. $\left[\left(\mathrm{Me}_{3} \mathrm{Si}\right)_{2} \mathrm{NMgC} \mathrm{CSiMe}_{3}(\mathrm{THF})\right]_{2} \quad$ (3) kristallisiert als Dimer in der Raumgruppe $P 2_{1} / \mathrm{c}$. Die Dimerisierung erfolgt über je ein verbrückendes Acetylid-Kohlenstoffatom. Das Dimer weist jedoch keine centrosymmetrische Struktur auf, da beide Acetylid-Liganden in Richtung auf ein und dasselbe Magnesiumatom $(\operatorname{Mg}(1))$ hin abgewinkelt sind (Abbildung 1). Die Mg-CBindungsabstände liegen zwischen 2.189(4) und 2.283(4) Å, wobei die Abstände zu $\operatorname{Mg}(2)$ generell etwas kürzer sind. Die $C \equiv C$ Abstände von 1.219(6) und 1.217(5) $\AA$ unterscheiden sich kaum von dem im freien Acetylen (1.20 ̊). Die Atome $\operatorname{Mg}(1)-C(1)-$ $\operatorname{Mg}(2)$ bzw. $\operatorname{Mg}(1)-C(6)-\operatorname{Mg}(2)$ bilden mit $87.04(16)^{\circ}$ und $88.03(15)^{\circ}$ jeweils einen nahezu idealen rechten Winkel. Die relativ kleinen Bindungswinkel $\operatorname{Mg}(1)-\mathrm{C}(1)-\mathrm{C}(2)$ 
$\left(100.7(3)^{\circ}\right)$ und $\operatorname{Mg}(1)-\mathrm{C}(6)-\mathrm{C}(7)\left(101.0(3)^{\circ}\right)$, bzw. $\mathrm{C}(1)-\mathrm{C}(2)-\operatorname{Si}(1)\left(171.3(4)^{\circ}\right)$ und $\mathrm{C}(6)-\mathrm{C}(7)-\mathrm{Si}(2)\left(170.9(4)^{\circ}\right)$ sprechen für Wechselwirkungen der acetylenischen $\pi$ Systeme mit dem Magnesiumatom $\mathrm{Mg}(1)$. Durch das Abwinkeln beider AcetylidLiganden in Richtung $\operatorname{Mg}(1)$ werden die Abstände $\operatorname{Mg}(1)-C(2)$ und $\operatorname{Mg}(1)-C(7)$ mit

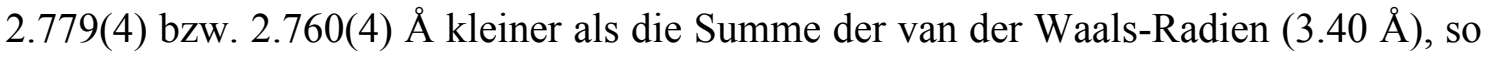
dass diese Liganden auch als 3-Elektronen-Donatoren aufgefasst werden können. Ähnliche Bindungsverhältnisse sind im trimeren heteroleptischen Komplex $\mathrm{Mg}_{3}(\mathrm{C} \equiv \mathrm{C}-\mathrm{t}$ $\mathrm{Bu})_{4}\left(\mathrm{~N}-i-\mathrm{Pr}_{2}\right)_{2}(\mathrm{THF})_{2}[20]$ und in $\left[\mathrm{MeC} \equiv \mathrm{CBe}(\mu-\mathrm{C} \equiv \mathrm{CMe})\left(\mathrm{NMe}_{3}\right)\right]_{2}$ beschrieben worden [21].

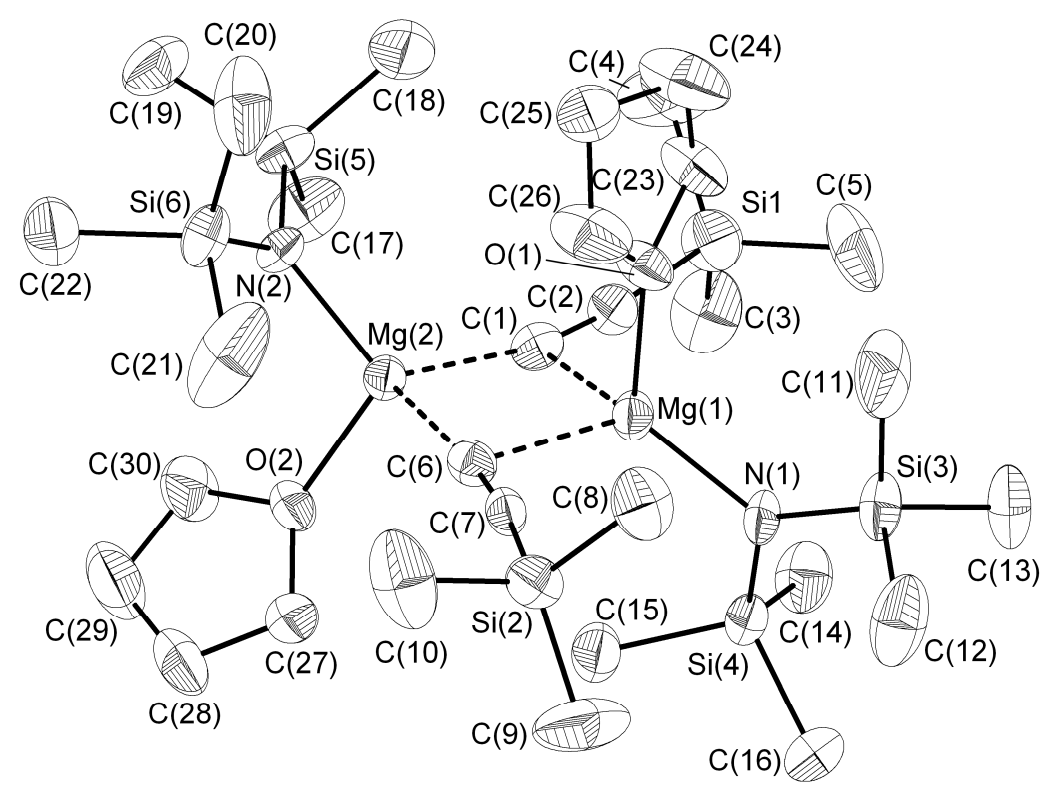

Abb. 1 Molekülstruktur von 3 ohne H-Atome und verwendete Atombezeichnungen. Die Ellipsoide umschreiben $50 \%$ Aufenthaltswahrscheinlichkeit. Ausgewählte Bindungslängen $[\AA]$ und -winkel $\left[^{\circ}\right]$ : $\mathrm{C}(1)-\mathrm{C}(2)$ 1.219(6), C(6)-C(7) 1.217(5), C(1)$\operatorname{Mg}(1)$ 2.283(4), C(1)-Mg(2) 2.202(5), C(6)-Mg(1) 2.255(4), C(6)-Mg(2) 2.189(4), C(2)$\mathrm{Mg}(1)$ 2.779(4), C(7)-Mg(1) 2.760(4), $\mathrm{Mg}(1)-\mathrm{N}(1)$ 1.989(3), $\mathrm{Mg}(1)-\mathrm{O}(1)$ 2.042(3), $\mathrm{Mg}(2)-\mathrm{N}(2)$ 2.002(3), $\mathrm{Mg}(2)-\mathrm{O}(2) \quad 2.032(3) ; \mathrm{C}(7)-\mathrm{C}(6)-\mathrm{Mg}(2)$ 170.8(4), C(2)-C(1)$\operatorname{Mg}(2)$ 167.9(3), N(1)-Mg(1)-C(6) 123.59(16), O(1)-Mg(1)-C(6) 94.58(14), N(1)-Mg(1)- 
$\mathrm{C}(1)$ 123.56(15), O(1)-Mg(1)-C(1) 94.59(14), C(6)-Mg(1)-C(1) 90.15(17), N(1)-Mg(1)$\mathrm{C}(7) \quad 103.73(14), \quad \mathrm{O}(1)-\mathrm{Mg}(1)-\mathrm{C}(7)$ 94.17(12), N(2)-Mg(2)-O(2) 106.43(14), N(2)$\mathrm{Mg}(2)-\mathrm{C}(6)$ 124.86(15), O(2)-Mg(2)-C(6) 99.92(14), N(2)-Mg(2)-C(1) 117.10(15), $\mathrm{O}(2)-\mathrm{Mg}(2)-\mathrm{C}(1) \quad 113.35(14), \quad \mathrm{C}(6)-\mathrm{Mg}(2)-\mathrm{C}(1) \quad 94.05(15), \quad \mathrm{Mg}(1)-\mathrm{C}(1)-\mathrm{Mg}(2)$ 87.04(16), $\mathrm{Mg}(1)-\mathrm{C}(6)-\mathrm{Mg}(2)$ 88.03(15), C(1)-C(2)-Si(1) 171.3(4), C(6)-C(7)-Si(2) 170.9(4).

$\left[\left(\mathrm{C}_{5} \mathrm{HMe}_{4}\right) \mathrm{MgC} \equiv \mathrm{CPh}(\mathrm{THF})\right]_{2}(\mathbf{5})$ kristallisiert als centrosymmetrisches Dimer in der Raumgruppe $P 2_{1} / \mathrm{c}$, wobei sich das Inversionszentrum in der Mitte des $\mathrm{Mg}_{2} \mathrm{C}_{2}$-Ringes befindet. Die Phenylacetylid-Liganden fungieren als Brückenbildner zwischen den beiden verzerrt tetraedrisch koordinierten Magnesiumatomen (Abbildung 2).

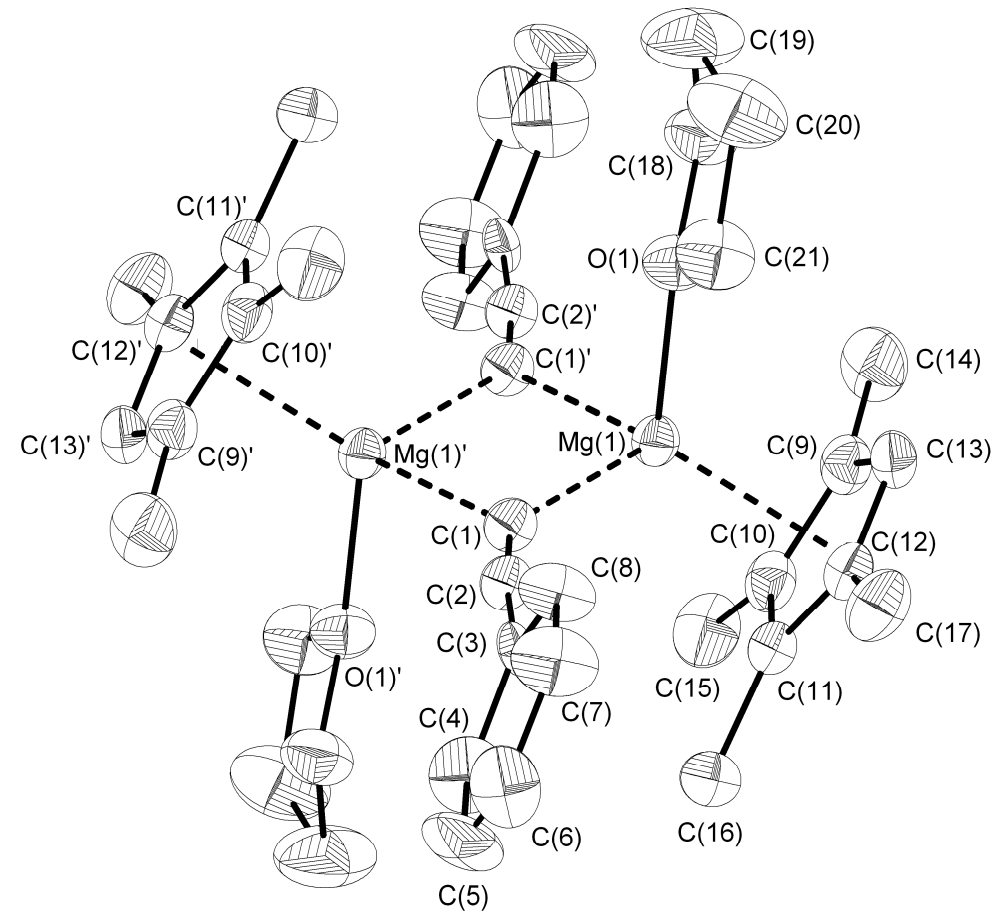

Abb. 2 Molekülstruktur von 5 ohne H-Atome und verwendete Atombezeichnungen. Die Ellipsoide umschreiben $50 \%$ Aufenthaltswahrscheinlichkeit. Ausgewählte Bindungslängen $[\AA \AA]$ und -winkel $\left[{ }^{\circ}\right]$ : $\mathrm{Mg}(1)$-centroid 2.087(1), C(1)-C(2) 1.210(2), $\mathrm{C}(1)-\mathrm{Mg}(1)$ 2.2177(18), C(1)-Mg(1)’ 2.2349(18), C(9)-Mg(1) 2.3920(18), C(10)-Mg(1) 2.3987(18), $\quad \mathrm{C}(11)-\mathrm{Mg}(1) \quad 2.4215(19), \quad \mathrm{C}(12)-\mathrm{Mg}(1) \quad 2.4275(18), \quad \mathrm{C}(13)-\mathrm{Mg}(1)$ 
2.4037(17), $\quad \mathrm{O}(1)-\mathrm{Mg}(1) \quad 2.0592(14) ; \quad \mathrm{C}(1)-\mathrm{C}(2)-\mathrm{C}(3) \quad 173.9(6), \quad \mathrm{C}(1)-\mathrm{Mg}(1)-\mathrm{C}(1){ }^{\prime}$ 96.76(6), $\mathrm{Mg}(1)-\mathrm{C}(1)-\mathrm{Mg}(1)^{\prime}$ 83.24(6), $\mathrm{O}(1)-\mathrm{Mg}(1)-\mathrm{C}(1)$ 95.34(6), $\mathrm{O}(1)-\mathrm{Mg}(1)-\mathrm{C}(1){ }^{\prime}$ 95.58(6), $\quad \operatorname{Mg}(1)-\mathrm{C}(1)-\mathrm{C}(2) \quad 140.76(14), \quad \operatorname{Mg}\left(1^{\prime}\right)-\mathrm{C}(1)-\mathrm{C}(2) \quad$ 136.00(4). Symmetrietransformationen um äquivalente Atome zu generieren: $-\mathrm{x},-\mathrm{y},-\mathrm{z}$.

Der Acetylid-Ligand ist nahezu linear ausgerichtet $\left(\mathrm{C}(1)-\mathrm{C}(2)-\mathrm{C}(3)=173.9(6)^{\circ}\right)$. Die Länge der $\mathrm{C}(1)-\mathrm{C}(2)$ Bindung liegt mit 1.210(2) $\AA$ im Bereich normaler Dreifachbindungen (1.20 Å). Die Bindungswinkel $\operatorname{Mg}(1)-C(1)-C(2)$ und $\operatorname{Mg}\left(1^{\prime}\right)-C(1)-$ $\mathrm{C}(2)$ sind mit $140.76(14)^{\circ}$ bzw. $136.00(14)^{\circ}$ nahezu identisch. Im Gegensatz dazu sind im analogen, unsubstituierten Cyclopentadienyl-Komplex $\left[\mathrm{C}_{5} \mathrm{H}_{5} \mathrm{Mg}(\mu-\mathrm{C} \equiv \mathrm{CPh})(\mathrm{THF})_{2}\right]_{2}$ [19] die Acetylid-Liganden unterschiedlich abgewinkelt (161.2(2) und 110.9(2) ${ }^{\circ}$, was im Sinne von $\sigma$-Donor/ $\pi$-Acceptor-Bindungen der verbrückenden Kohlenstoffatome gedeutet wurde. Die beiden Komplexe unterscheiden sich auch hinsichtlich der Anordnung der Phenylreste. Während diese in $\left[\mathrm{C}_{5} \mathrm{H}_{5} \mathrm{Mg}(\mu-\mathrm{C} \equiv \mathrm{CPh})(\mathrm{THF})_{2}\right]_{2}$ gegeneinander verdrillt sind [19], ordnen sie sich in 5 mit den sterisch anspruchsvolleren Tetramethylcyclopentadienyl-Liganden parallel zueinander an. Die Bindungslängen $\mathrm{Mg}-\mathrm{C}(1)$ und $\mathrm{Mg}\left(1^{\prime}\right)-\mathrm{C}(1)$ unterscheiden sich mit 2.177(18) und 2.2349(18) Å nur geringfügig von jenen in $\left[\mathrm{C}_{5} \mathrm{H}_{5} \mathrm{Mg}(\mu-\mathrm{C} \equiv \mathrm{CPh})(\mathrm{THF})_{2}\right]_{2}(2.18 .5(2)$ und 2.277(3) Å) [19]. Die Cyclopentadienylringe in 5 sind mit einem Bindungsabstand von 2.087(1) $\AA$ (Centroid) an den Magnesiumatomen gebunden. Trotz des geringeren sterischen Anspruchs der unsubstituierten Cyclopentadienylringe sind in $\left[\mathrm{C}_{5} \mathrm{H}_{5} \mathrm{Mg}(\mu-\right.$ $\left.\mathrm{C} \equiv \mathrm{CPh})(\mathrm{THF})_{2}\right]_{2}$ längere Metall-Centroid-Abstände (2.120(5) bis 2.133(6) $\AA$ ) zu finden [19]. 
$\mathrm{C}_{5} \mathrm{HMe}_{4} \mathrm{MgC} \equiv \mathrm{CSiMe}_{3}(\mathrm{THF})_{2}$ (6) kristallisiert aus Toluol als Monomer in der Raumgruppe $P 2_{1} / \mathrm{n}$. Das Magnesiumatom ist auch hier vierfach koordiniert. Aufgrund des vergleichsweise großen Raumanspruchs des Tetramethylcyclopentadienyl-Liganden werden die drei übrigen Liganden gemäß den Centroid-Mg-C(1)/O(1)/O(2) Bindungswinkeln von $124.86(13) / 117.35(8) / 122.29(8)^{\circ}$ aus den idealen Tetraederpositionen gedrängt (Abbildung 3).

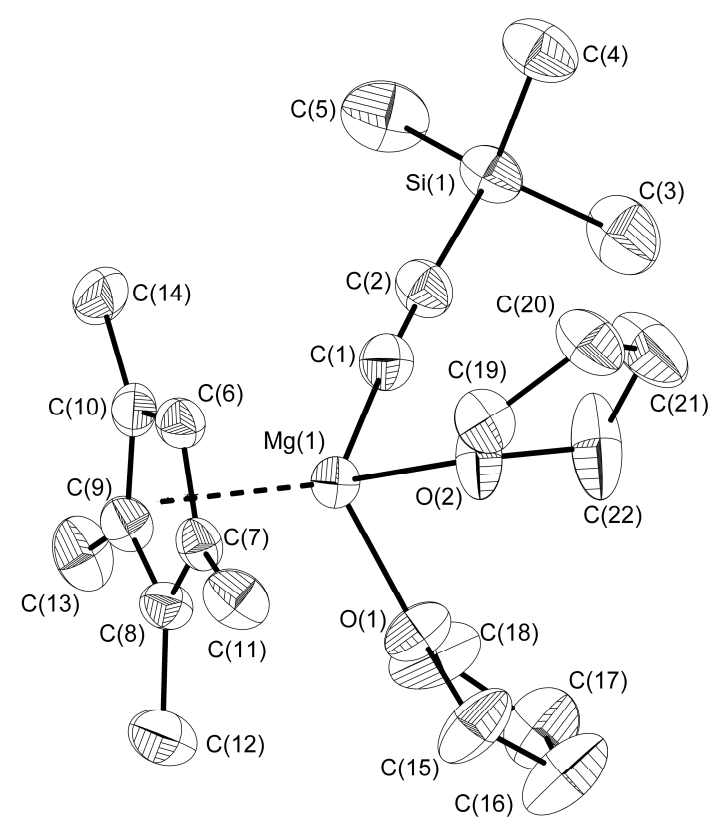

Abb. 3 Molekülstruktur von 6 ohne H-Atome und verwendete Atombezeichnungen. Die Ellipsoide umschreiben $50 \%$ Aufenthaltswahrscheinlichkeit. Ausgewählte Bindungslängen $[\AA]$ und -winkel [ $\left.{ }^{\circ}\right]$ : Centroid-Mg(1) 2.119(1), C(1)-Mg(1) 2.135(4), $\mathrm{C}(1)-\mathrm{C}(2)$ 1.211(5), C(6)-Mg(1) 2.407(3), C(7)-Mg(1) 2.442(3), C(8)-Mg(1) 2.451(4), $\mathrm{C}(9)-\mathrm{Mg}(1) \quad 2.438(3) \quad \mathrm{Mg}(1)-\mathrm{O}(2) \quad 2.068(3), \mathrm{Mg}(1)-\mathrm{O}(1) \quad 2.077(3) ; \mathrm{C}(2)-\mathrm{C}(1)-\mathrm{Mg}(1)$ 172.4(3), $\mathrm{O}(2)-\mathrm{Mg}(1)-\mathrm{O}(1)$ 88.28(11), O(2)-Mg(1)-C(1) 98.01(13), O(1)-Mg(1)-C(1) 97.98(12), Centroid-Mg(1)-C(1) 124.86(13), Centroid-Mg(1)-O(1) 117.35(8), Centroid$\operatorname{Mg}(1)-\mathrm{O}(2) 122.29(8)$ 
Die Bindungslänge Mg-C(1) von 2.135(4) Å unterscheidet sich nur wenig von jenen im dimeren Komplex 5 (2.2177(18) und 2.2349(18)). Die Länge der $\mathrm{C} \equiv \mathrm{C}$ Dreifachbindung entspricht mit 1.211(5) Å der Länge normaler Dreifachbindungen und die Mg-O(THF) Abstände liegen mit 2.068(3) und 2.077(3) Å im Bereich der für andere THF koordinierte Magnesiumkomplexe bestimmten Werte [22].

\section{Katalytische Aktivität von 3, 5, 6 und 7}

Der Magnesiumkomplex 3 katalysiert die Polymerisation von Methylmethacrylat in Toluol, nicht jedoch in THF als Lösemittel (Tabelle 1). Unter im Übrigen gleichen Reaktionsbedingungen polymerisiert bei $-78{ }^{\circ} \mathrm{C}$ deutlich mehr Monomer als bei Raumtemperatur (vergleiche Ansätze 1 und 2 bzw. 4 und 5). Die Polymerisationen führen schon nach kurzer Zeit zur Bildung viskoser Lösungen.

Auch Komplex 5 katalysiert in Toluol die Polymerisation von Methylmethacrylat, jedoch nur mit schlechten Ausbeuten (Tabelle 1, Ansätze 6 und 7). Im polaren Lösemittel THF steigert sich dagegen die Aktivität von 5 erheblich (Ansätze 8 - 11). So werden bei $-78{ }^{\circ} \mathrm{C}$ und in Gegenwart von $\mathrm{AlEt}_{3}$ als Cokatalysator quantitative Ausbeuten erzielt. Bei Raumtemperatur und ohne $\mathrm{AlEt}_{3}$ Zusatz sinkt die Aktivität des Katalysators beträchtlich ab, vor allem wegen dessen teilweiser thermischer Zersetzung.

Der Magnesiumkomplex 6 zeigt praktisch die gleichen katalytischen Eigenschaften wie Komplex 5. Während in Toluol nur schlechte Ausbeuten an PMMA mit relativ enger Molmassenverteilung $\mathrm{zu}$ erzielen sind, gelingt es in THF wieder nahezu quantitative Ausbeuten zu erhalten. 
Die Aktivität des Calciumkomplexes 7 wurde nur in THF untersucht. Auch hier werden in Anwesenheit des Cokatalysators $\mathrm{AlEt}_{3}$ bei $-78^{\circ} \mathrm{C}$ deutlich höhere Ausbeuten erzielt als dies bei Raumtemperatur der Fall ist. Wie die Ergebnisse der Ansätze 19 und 20 zeigen, ist bei Raumtemperatur die Ausbeute an Polimerisat in Gegenwart von $\mathrm{AlEt}_{3}$ als Cokatalysator um den Faktor 3.5 niedriger als ohne Cokatalysator.

Tabelle 1 Bedingungen und Ergebnisse der Polymerisation von Methylmethacrylat mit 3, 5, 6 und 7 (Polymerisationsdauer: 2h, D = Polydispersität, $\mathrm{M}_{\mathrm{n}}=$ Molmasse

\begin{tabular}{|l|l|l|l|l|l|l|l|l|}
\hline Ansatz & Kat. & Lösemittel & $\begin{array}{l}\mathrm{T} \\
{ }^{\circ} \mathrm{C}\end{array}$ & $\begin{array}{l}\text { Molverhältnis } \\
\text { Kat/Et } 3 \text { Al/MMA }\end{array}$ & $\begin{array}{l}\text { Ausbeute } \\
\%\end{array}$ & $\begin{array}{l}\mathrm{M}_{\mathrm{n}} \\
\mathrm{g} / \mathrm{mol}\end{array}$ & $\mathrm{D}$ & $\begin{array}{l}\mathrm{M}_{\mathrm{n}}(\mathrm{GPC}) / \\
\mathrm{M}_{\mathrm{n}} \text { (theor) }\end{array}$ \\
\hline 1 & $\mathbf{3}$ & Toluol & -78 & $1 / 3 / 110$ & 83 & 14.800 & 4.1 & 1.6 \\
2 & $\mathbf{3}$ & Toluol & +23 & $1 / 3 / 110$ & 22 & unlöslich & - & - \\
3 & $\mathbf{3}$ & Toluol & -78 & $1 / 9 / 340$ & 18 & 71.700 & 2.18 & 12 \\
4 & $\mathbf{3}$ & Toluol & -78 & $1 / 0 / 110$ & 19 & 30.800 & 5.02 & 15 \\
5 & $\mathbf{3}$ & Toluol & +23 & $1 / 0 / 110$ & 9 & 42.100 & 1.8 & 42 \\
6 & $\mathbf{5}$ & Toluol & -78 & $1 / 2 / 75$ & 55 & 545.000 & 2 & 132 \\
7 & $\mathbf{5}$ & Toluol & +23 & $1 / 2 / 100$ & 13 & 14.800 & 2.02 & 11.4 \\
8 & $\mathbf{5}$ & THF & -78 & $1 / 2 / 100$ & 100 & 19.800 & 2.03 & 1.9 \\
9 & $\mathbf{5}$ & THF & -78 & $1 / 5 / 220$ & 100 & 104.000 & 2.99 & 4.7 \\
10 & $\mathbf{5}$ & THF & -78 & $1 / 0 / 100$ & 96 & 324.000 & 1.94 & 45 \\
11 & $\mathbf{5}$ & THF & +23 & $1 / 0 / 100$ & 16 & 8.700 & 1.94 & 7.3 \\
12 & $\mathbf{6}$ & Toluol & -78 & $1 / 2 / 60$ & 26 & 9.600 & 1.32 & 1.6 \\
13 & $\mathbf{6}$ & Toluol & -78 & $1 / 2 / 110$ & 75 & 14.700 & 2.12 & 1.4 \\
14 & $\mathbf{6}$ & Toluol & -78 & $1 / 9 / 870$ & 12 & 19.000 & 1.43 & 0.2 \\
15 & $\mathbf{6}$ & Toluol & +23 & $1 / 0 / 90$ & 31 & 13.400 & 3.93 & 1.5 \\
16 & $\mathbf{6}$ & THF & -78 & $1 / 2 / 110$ & 99 & 204.400 & 2.08 & 19.3 \\
17 & $\mathbf{6}$ & THF & -78 & $1 / 6 / 280$ & 97 & 230.000 & 3.23 & 8.3 \\
18 & $\mathbf{7}$ & THF & -78 & $1 / 2 / 100$ & 92 & 32.700 & 1.83 & 3.6 \\
19 & $\mathbf{7}$ & THF & +23 & $1 / 2 / 100$ & 10 & 12.300 & 1.33 & 12.5 \\
20 & $\mathbf{7}$ & THF & +23 & $1 / 0 / 100$ & 35 & 18.500 & 1.42 & 5.3 \\
\hline
\end{tabular}


Die mit allen vier Katalysatoren erhaltenen Polymere sind syndiotaktisch [13]. Auf diese Eigenschaft der Polymeren soll im Zusammenhang mit den Ergebnissen, die bei der Polymerisation anderer polarer Monomere wie $\varepsilon$-Caprolakton und L-Laktid mit gleichfalls anderen Erdalkalimetall-Katalysatoren erhalten wurden in einer weiteren Publikation ausführlich eingegangen werden [23].

\section{Experimenteller Teil}

Alle Arbeiten wurden mit sorgfältig getrockneten und entgasten Lösemitteln in Schlenkkolben in einer Atmosphäre von gereinigtem Stickstoff durchgeführt. $\mathrm{HC} \equiv \mathrm{CPh}$, $\mathrm{HC} \equiv \mathrm{CSiMe}_{3}$ und $\mathrm{MgBu}_{2}$ wurden von Aldrich bezogen, $\mathrm{Mg}\left[\mathrm{N}\left(\mathrm{SiMe}_{3}\right)_{2}\right]_{2}(\mathrm{THF})_{2}$ [25] nach entsprechender Literaturvorschrift hergestellt. $-{ }^{1} \mathrm{H}-$ und ${ }^{13} \mathrm{C}-\mathrm{NMR}$ : Bruker ARX 200 (200 bzw. $50.32 \mathrm{MHz}$ ) gegen TMS. -Elementaranalysen: Perkin-Elmer 240C CHNS/O Analyzer.

$\left[\left(\mathbf{M e}_{3} \mathbf{S i}\right)_{2} \mathbf{N M g C} \equiv \mathbf{C P h}(\mathbf{T H F})\right]_{2} \quad$ (2): $\mathrm{Zu}$ einer Lösung von $0.77 \mathrm{~g}$ (2.23 mmol) $\mathrm{Mg}\left[\mathrm{N}\left(\mathrm{SiMe}_{3}\right)_{2}\right]_{2}(\mathrm{THF})_{2}(\mathbf{1})$ in $10 \mathrm{~mL}$ THF werden $0.23 \mathrm{~g}(2.27 \mathrm{mmol}) \mathrm{PhC} \equiv \mathrm{CH}$ getropft und die Reaktionsmischung zunächst $2 \mathrm{~h}$ bei Raumtemperatur, dann $5 \mathrm{~h}$ bei $80{ }^{\circ} \mathrm{C}$ gerührt und anschließend langsam abgekühlt. Nach Entfernung der flüchtigen Bestandteile bei $10^{-2}$ mbar erhält man ein viskoses Öl, das in Hexan aufgenommen wird. Aus der auf $-30{ }^{\circ} \mathrm{C}$ abgekühlten Lösung fällt im Verlauf mehrerer Stunden 2 als farbloser Feststoff aus. Ausbeute: $0.69 \mathrm{~g}(86 \%) . \mathrm{C}_{18} \mathrm{H}_{31} \mathrm{MgNOSi}_{2}$ (357.92): ber. $\mathrm{C}$ 60.40, H 8.73, N 3.91; gef. C 59.92, H 8.79, N $3.52 \%$. 
${ }^{1} \mathbf{H}$ NMR $\left(200 \mathrm{MHz}, \mathrm{C}_{6} \mathrm{D}_{6}\right): \delta=0.04\left(\mathrm{~s}, 18 \mathrm{H}, \mathrm{SiMe}_{3}\right), 1.77$ (m, $\left.4 \mathrm{H}, \mathrm{THF}\right), 3.62(\mathrm{~m}, 4$ H, THF), $7.00-7.23(\mathrm{~m}, 5 \mathrm{H}, \mathrm{Ph})$.

$\left[\left(\mathrm{Me}_{3} \mathbf{S i}\right)_{2} \mathbf{N M g C} \equiv \mathrm{CSiMe}_{3}(\mathbf{T H F})\right]_{2}$ (3): Zu einer Lösung von $0.68 \mathrm{~g}(1.4 \mathrm{mmol}) \mathbf{1}$ in 5 $\mathrm{mL}$ THF werden $0.15 \mathrm{~g}(1.5 \mathrm{mmol}) \quad \mathrm{Me}_{3} \mathrm{SiC} \equiv \mathrm{CH}$ bei $-78 \quad{ }^{\circ} \mathrm{C}$ getropft. Die Reaktionsmischung wird langsam erwärmt und $6 \mathrm{~h}$ bei $80^{\circ} \mathrm{C}$ unter Rückfluss erhitzt. Nach Entfernung der flüchtigen Bestandteile bei $10^{-2}$ mbar erhält man ein farbloses Öl, das in Hexan gelöst wird. Aus der auf $-30{ }^{\circ} \mathrm{C}$ abgekühlten Lösung kristallisiert im Verlauf mehrerer Wochen 3 in Form farbloser Nadeln. Ausbeute: 0.36 g (36 \%). $\mathrm{C}_{15} \mathrm{H}_{35} \mathrm{MgNOSi}_{3}$ (354.01): ber. C 50.89, H 9.97, N 3.96; gef. C 50.36, H 9.72, N 3.62 $\%$.

${ }^{1} \mathbf{H}$ NMR $\left(200 \mathrm{MHz}, \mathrm{C}_{6} \mathrm{D}_{6}\right): \delta=0.21\left(\mathrm{~s}, 9 \mathrm{H}, \mathrm{SiMe}_{3}\right), 0.34\left(\mathrm{~s}, 18 \mathrm{H}, \mathrm{SiMe}_{3}\right), 1.41(\mathrm{~m}, 4$ H, THF), 3.93 (m, $4 \mathrm{H}, \mathrm{THF}) .-{ }^{13} \mathbf{C} \mathbf{N M R}\left(50,32 \mathrm{MHz}, \mathrm{C}_{6} \mathrm{D}_{6}\right): \delta=0.27\left(\mathrm{SiMe}_{3}\right), 25.09$ (THF), 70.02 (THF), $134.14(\mathrm{C} \equiv \mathrm{C}), 143.79(\mathrm{C} \equiv \mathrm{C})$.

$\left[\left(\mathbf{C}_{5} \mathbf{H M e}_{4}\right) \mathbf{M g C} \equiv \mathbf{C P h}(\mathbf{T H F})\right]_{2} \quad$ (5): $\mathrm{Zu} \quad$ einer $\quad$ Lösung $\quad$ von 0.54 g $\quad(2 \mathrm{mmol})$ $\mathrm{Mg}\left(\mathrm{C}_{5} \mathrm{HMe}_{4}\right)_{2}$ in $10 \mathrm{~mL}$ THF werden unter Rühren bei Raumtemperatur $2 \mathrm{~mL}$ einer $1 \mathrm{M}$ Lösung von $\mathrm{MgBu}_{2}$ in Heptan getropft und $2 \mathrm{~h}$ gerührt. Die Lösung von entstandenem 4 wird dann auf $-78{ }^{\circ} \mathrm{C}$ gekühlt und mit $0.41 \mathrm{~g}(4 \mathrm{mmol}) \mathrm{PhC} \equiv \mathrm{CH}$ versetzt. Die Reaktionsmischung wird unter Rühren langsam auf Raumtemperatur erwärmt und weitere $2 \mathrm{~h}$ gerührt. Anschließend werden die flüchtigen Bestandteile im Vakuum entfernt. Das zurückbleibende farblose 5 wird mehrmals mit Hexan gewaschen, bei $10^{-2}$ mbar getrocknet und abschließend aus Toluol umkristallisiert. Ausbeute: $1.22 \mathrm{~g}$ (96 \%). 
- Schmp. $179.3{ }^{\circ} \mathrm{C}$ (Zers.). $\mathrm{C}_{21} \mathrm{H}_{26} \mathrm{MgO}$ (318.74): ber. C 79.13, H 8.22; gef. C 78.17, $\mathrm{H}$ $7.89 \%$.

${ }^{1}$ H NMR $\left(200 \mathrm{MHz}, \mathrm{C}_{4} \mathrm{D}_{8} \mathrm{O}\right): \delta=1.81(\mathrm{~m}, 4 \mathrm{H}, \mathrm{THF}), 2.04\left(\mathrm{~s}, 6 \mathrm{H}, \mathrm{CH}_{3}\right), 2.09(\mathrm{~s}, 6 \mathrm{H}$, $\left.\mathrm{CH}_{3}\right), 3.67$ (m, $\left.4 \mathrm{H}, \mathrm{THF}\right), 5.39\left(\mathrm{~s}, 1 \mathrm{H}, \mathrm{C}_{5} \mathrm{H}\right), 7.00-7.25(\mathrm{~m}, 5 \mathrm{H}, \mathrm{Ph}) .-{ }^{13} \mathbf{C} \mathbf{N M R}$ $\left(100,64 \mathrm{MHz}, \mathrm{C}_{4} \mathrm{D}_{8} \mathrm{O}\right): \delta=11.4\left(\mathrm{CH}_{3}\right), 13.5\left(\mathrm{CH}_{3}\right), 25.3$ (THF), 67.4 (THF), 100.9 $\left(\mathrm{CH}_{\mathrm{Cp}}\right), 109.4\left(\mathrm{CMe}_{\mathrm{Cp}}\right), 111.9\left(\mathrm{CMe}_{\mathrm{Cp}}\right), 125.0\left(\mathrm{Ph}-\mathrm{C}_{\mathrm{i}}\right), 128.1\left(\mathrm{Ph}-\mathrm{C}_{\mathrm{o}}\right), 131.9\left(\mathrm{Ph}-\mathrm{C}_{\mathrm{p}}\right)$.

$\left(\mathbf{C}_{5} \mathbf{H M e}_{4}\right) \mathbf{M g C} \equiv \mathrm{CSSiMe}_{3}(\mathbf{T H F})_{2} \quad$ (6): $\mathrm{Zu}$ einer Lösung von 0.54 g (2 mmol) $\operatorname{Mg}\left(\mathrm{C}_{5} \mathrm{HMe}_{4}\right)_{2}$ in $10 \mathrm{~mL}$ THF werden unter Rühren bei Raumtemperatur $2 \mathrm{~mL}$ einer $1 \mathrm{M}$ Lösung von $\mathrm{MgBu}_{2}$ in Heptan getropft und $2 \mathrm{~h}$ gerührt. $\mathrm{Zu}$ díeser Lösung von entstandenem 4 werden nach $2 \mathrm{~h}$ bei $-78{ }^{\circ} \mathrm{C} 0.39 \mathrm{~g}$ ( $\left.4 \mathrm{mmol}\right) \mathrm{Me}_{3} \mathrm{SiC} \equiv \mathrm{CH}$ gegeben und die Reaktionsmischung unter Rühren langsam auf Raumtemperatur erwärmt. Nach $12 \mathrm{~h}$ Rühren bei Raumtemperatur und $4 \mathrm{~h}$ Rühren bei $60{ }^{\circ} \mathrm{C}$ werden die flüchtigen Bestandteile bei Raumtemperatur im Vakuum entfernt. Das verbleibende rohe 6 wird mehrmals mit Hexan gewaschen und anschließend bei $10^{-2}$ mbar getrocknet. Durch Kristallisation aus Toluol wird reines $\mathbf{6}$ in Form farbloser, rhombischer Kristalle erhalten. Ausbeute: $1.08 \mathrm{~g}$ (70 \%). - Schmp. $>320{ }^{\circ} \mathrm{C}$ (Zers.). $\mathrm{C}_{22} \mathrm{H}_{38} \mathrm{MgO}_{2} \mathrm{Si}$ (386.92): ber. C 68.29, H 9.90; gef. C 68.89, H 9.36\%.

${ }^{1}$ H NMR $\left(200 \mathrm{MHz}, \mathrm{C}_{6} \mathrm{D}_{6}\right): \delta=0.28\left(\mathrm{~s}, 9 \mathrm{H}, \mathrm{SiMe}_{3}\right), 1.43$ (m, $\left.4 \mathrm{H}, \mathrm{THF}\right), 2.17$ (s, $6 \mathrm{H}$, Cp- $\left.\mathrm{CH}_{3}\right), 2.28\left(\mathrm{~s}, 6 \mathrm{H}, \mathrm{Cp}-\mathrm{CH}_{3}\right), 3.79$ (m, $\left.4 \mathrm{H}, \mathrm{THF}\right), 5.64$ (s, $\left.1 \mathrm{H}, \mathrm{C}_{5} \mathrm{H}\right) .-{ }^{13} \mathrm{C} \mathrm{NMR}$ $\left(50,32 \mathrm{MHz}, \mathrm{C}_{6} \mathrm{D}_{6}\right): \delta=0.89\left(\mathrm{SiMe}_{3}\right), 11.84\left(\mathrm{Cp}-\mathrm{CH}_{3}\right), 14.24\left(\mathrm{Cp}-\mathrm{CH}_{3}\right), 25.25(\mathrm{THF})$, 69.19 (THF), $103.27\left(\mathrm{CH}_{\mathrm{Cp}}\right), 110.21\left(\mathrm{CMe}_{\mathrm{Cp}}\right), 111.06\left(\mathrm{CMe}_{\mathrm{Cp}}\right)$. 
$\left[\left(\mathbf{C}_{5} \mathbf{H M e}_{4}\right) \mathbf{C a C} \equiv \mathbf{C P h}(\mathbf{T H F})\right]_{2} \quad \mathbf{( 7 ) :} \mathrm{Zu}$ einer Lösung $\quad$ von 1 g $\quad(8 \mathrm{mmol})$ Tetramethylcyclopentadien und $0.82 \mathrm{~g}(8 \mathrm{mmol}) \mathrm{PhC} \equiv \mathrm{CH}$ in $40 \mathrm{~mL}$ THF werden $0.32 \mathrm{~g}$ (8 mmol) Calcium-Stückchen gegeben. In die auf $-40{ }^{\circ} \mathrm{C}$ gekühlte Mischung wird so lange $\mathrm{NH}_{3}$ eingeleitet bis alles Calcium gelöst ist. Anschließend wird noch $2 \mathrm{~h}$ bei -30 ${ }^{\circ} \mathrm{C}$ und dann über Nacht bei Raumtemperatur gerührt, wobei sich die zunächst entstandene rosa Suspension weiß färbt. Nach Filtration, Waschen des Rückstandes mit Pentan und dreimaligem Extrahieren des verbleibenden Pulvers mit je $20 \mathrm{~mL}$ Toluol bleibt 9 als weißer, luftempfindlicher Feststoff zurück. Ausbeute: 0.87 g (38\%). $\mathrm{C}_{17} \mathrm{H}_{18} \mathrm{Ca}(\mathrm{THF})_{0.4}$ (291.2): ber. C 76.70, H 7.34; gef. C 70.52, H $6.81 \%$.

${ }^{1}$ H NMR $\left(200 \mathrm{MHz}, \mathrm{C}_{5} \mathrm{D}_{5} \mathrm{~N}\right): \delta=1.59(\mathrm{~m}, 1.4 \mathrm{H}, \mathrm{THF}), 1.78\left(\mathrm{~s}, 6 \mathrm{H}, \mathrm{CH}_{3}\right), 2.17(\mathrm{~s}, 6$ $\left.\mathrm{H}, \mathrm{CH}_{3}\right), 3.64(\mathrm{~m}, 1.4 \mathrm{H}, \mathrm{THF}), 5.75\left(\mathrm{~s}, 1 \mathrm{H}, \mathrm{C}_{5} \mathrm{H}\right), 7.18(\mathrm{~s}, 1 \mathrm{H}, \mathrm{Ph}), 7.34\left(\mathrm{t},{ }^{3} J(\mathrm{HH})=\right.$ $7.4 \mathrm{~Hz}, 2 \mathrm{H}, \mathrm{Ph}), 7.89\left(\mathrm{t},{ }^{3} J(\mathrm{HH})=7.4 \mathrm{~Hz}, 2 \mathrm{H}, \mathrm{Ph}\right)$.

\section{Röntgenstrukturanalysen von 3, 5 und 6}

Die kristallographischen Daten und die Messparameter sind in Tabelle 2 zusammengefasst. Sie wurden mit einem Siemens SMART CCD Diffraktometer unter Verwendung von $\mathrm{MoK}_{\alpha}$ Strahlung (Graphitmonochromator) bei $173 \mathrm{~K}$ erhalten. Es wurden empirische Absorptionskorrekturen (SADABS) [25] durchgeführt. Die Strukturlösung erfolgte mit direkten Methoden unter Verwendung von SHELXS-97 [26], die Verfeinerung mit SHELXL-97 [27]. Alle Nicht-Wasserstoffatome wurden anisotrop verfeinert. Die Wasserstoffatome wurden in idealisierten Positionen berechnet. CCDC-722420 (3), CCDC-722421 (5) und CCDC-722422 (6) enthalten die 
beim Cambridge Crystallographic Data Centre hinterlegten Kristallstrukturdaten. Anforderungen: www.ccdc.cam.ac.uk/data_request/cif.

\section{Tabelle 2}

\section{Polymerisationen}

Die jeweils zur Anwendung kommenden Mengen an Monomer, Lösemittel und gegebenenfalls Triethylaluminium wurden mit Spritzen in einen Schlenckkolben eingefüllt und auf die gewünschte Temperatur gebracht. Die in einer Glovebox aufbewahrten, als Kristalle oder in Pulverform vorliegenden Katalysatoren wurden in dieser Schutzgasatmosphäre abgewogen, in einem Schlenckkolben mit dem entsprechenden Lösemittel versetzt und diese Lösung unter starkem Rühren zu dem, das Monomer enthaltende Gemisch gegeben. Nach vorbestimmter Zeit wurde die Polymerisation durch Zugabe einer geringen Menge eines Methanol/HCl Gemisches abgebrochen. Durch Hinzufügen großer Mengen Methanol zur Reaktionslösung (v/v 10:1) wurde das Polymer ausgefällt, dieses anschließend abfiltriert und dann mehrere Stunden im Vakuum $\left(10^{-2}\right.$ bar $)$ getrocknet.

Die GPC-Analytik erfolgte mit der Anlage 150C der Firma Waters unter Verwendung einer mixedbed-Säule (PLgel $5 \mu \mathrm{m}$-Mixed-C, Polymer Laboratories) bei Raumtemperatur. Die Säule wurde mit PMMA-Standards mit Molmassen zwischen 3.000 und 4.000.000 g/mol der Firmen PSS und Polyscience unter Anwendung der PSS GPC-Kalibration V3.11 Software kalibriert. Als Eluent diente über $\mathrm{SnCl}_{2} / \mathrm{KOH}$ destilliertes THF, dessen Flussrate auf $1 \mathrm{~mL} / \mathrm{min}$ eingestellt wurde. PolymerKonzentrationen wurde über einen Refraktionsindex (RI)-Detektor, Modell Optilab DSP der Firma Wyatt Technology bei einer Wellenlänge von $633 \mathrm{~nm}$ bestimmt. Alle Proben 
wurden in THF mit 0.1 Vol\% Toluol als innerem Standard gelöst und durch $45 \mu \mathrm{m}$ Spritzenfilter filtriert, um Schwebepartikel zu entfernen. In einigen Fällen mit sichtbaren Verunreinigungen wurde die Probe zunächst durch Filtration über ein Kieselbett geklärt. Die Erfassung und Auswertung der Daten erfolgte mit der Software WinGPC 4 der Firma PSS.

\section{Dank}

Wir danken dem Fonds der Chemischen Industrie und der Deutschen Forschungsgemeinschaft für finanzielle Unterstützung dieser Arbeit

\section{Literatur}

[1] P. U. Rokkanen, O. Böstman, E. Hirvensalo, E. A. Mäkelä, E. K. Partio, H. Pätiälä, S. Vainionpää, K. Vihtonen, P. Törmälä, Biomaterials 2001, 21, 2607.

[2] a) B. Francois, C. Mathis, Makromol. Chem. 1972, 156, 7; b) B. Francois, C. Mathis, Makromol. Chem. 1972, 156, 17.

[3] H. R. Kricheldorf, A. Serrsa, Polymer Bull. 1985, 14, 497.

[4] K. A. Allen, B. G. Gowenlock, W. E. Lindsell, J. Polym, Sci.: Polym. Chem. 1974, 12, 1131.

[5] Y. Li, H. Deng, W. Brittain, M. S. Chisholm, Polymer Bull. 1999, 42, 635.

[6] W. E. Lindsell, F. C. Robertson, I. Soutar, D. H. Richards, Eur. Polymer J. 1981, $17,107$.

[7] Z. Zhong, P. J. Dijkstra, C. Birg, M. Westerhausen, J. Feijen, Macromolecules 2001, 34, 3863. 
[8] Z. Zhong, M. J. K. Ankoné, P. J. Dijkstra, C. Birg, M. Westerhausen, J. Feijen, Polymer Bull. 2001, 46, 51.

[9] Z. Tang, X. Chen, Q. Liang, X. Bian, L. Yang, L. Piao, X. Jing, J. Polymer Sci.: Polymer Chem. 2003, 41, 1934.

[10] P. Dobrzynski, J. Kasperczyk, M. Bero, Makromolecules 1999, 32, 4735.

[11] a) S. Harder, F. Feil, K. Knoll, Angew. Chem. 2001, 113, 4391; Angew. Chem. Int. Ed. 2001, 40, 4261; b) S. Harder, F. Feil, A. Weeber, Organometallics 2001, 20 , 1044; c) S. Harder, F. Feil, Organometallics 2002, 221, 2268; d) F. Feil, S. Harder, Eur. J. Inorg. Chem. 2003, 3401.

[12] H. Schumann, J. Gottfriedsen, M. Glanz, S. Dechert, J. Demtschuk, J. Organomet. Chem. 2001, 617, 588.

[13] A. Steffens, Dissertation Technische Universität Berlin 2005.

[14] A. Steffens, H. Schumann, Macromol. Symp. 2006, 236, 203.

[15] M. J. McCormick, S. C. Sockwell, C. E. H. Davis, T. P. Hanusa, J. C. Huffmann, Organometallics 1989, 8, 2044.

[16] M. J. Harvey, T. P. Hanusa, Organometallics 2000, 19, 1556.

[17] D. J. Burkey, E. K. Alexander, T. P. Hanusa, Organometallics 1994, 13, 2773.

[18] D. J. Burkey, T. P. Hanusa, Organometallics 1996, 15, 4971.

[19] A. Xia, M. J. Heeg, C. H. Winter, Organometallics 2003, 22, 1793.

[20] K. C. Yang, C. C. Chang, J. Y. Huang, C. C. Lin, G. H. Lee, Y. Wang, M. Y. Chiang, J. Organomet. Chem. 2002, 648, 176.

[21] M. Save, M. Schappacher, A. Soum, Macromol. Chem. Phys. 2002, 203, 889.

[22] M. A. G. M. Tinga, G. Schat, O. S. Akkerman, F. Bickelhaupt, E. Horn, H. Kooijman, W. J. J. Smeets, A. L. Spek, J. Am. Chem. Soc. 1993, 115, 2808. 
[23] A. Steffens, J. Springer, H. Schumann, Publikation in Vorbereitung.

[24] D. C. Bradley, M. B. Hursthouse, A. A. Ibrahim, K. M. A. Malik, M. Motevalli, R. Möseler, H. Powell, J. D. Runnacles, A. C. Sullivan, Polyhedron 1990, 9, 2959.

[25] G. M. Sheldrick, SADABS Program for Empirical Absorption Correction of Area Detector Data, Universität Göttingen, Göttingen, Germany 1996.

[26] G. M. Sheldrick, SHELXS-97 Program for the Solution of Crystal Structures, Universität Göttingen, Göttingen, Germany 1998.

[27] G. M. Sheldrick, SHELXL-97 Program for the Refinement of Crystal Structures, Universität Göttingen, Göttingen, Germany 1998. 
Tabelle 2 Kristall- und Strukturverfeinerungsdaten für 3, 5 und 6.

\begin{tabular}{|c|c|c|c|}
\hline & 3 & 5 & 6 \\
\hline Empirische Formel & $\mathrm{C}_{30} \mathrm{H}_{70} \mathrm{Mg}_{2} \mathrm{~N}_{2} \mathrm{O}_{2} \mathrm{Si}_{6}$ & $\mathrm{C}_{42} \mathrm{H}_{52} \mathrm{Mg}_{2} \mathrm{O}_{2}$ & $\mathrm{C}_{22} \mathrm{H}_{38} \mathrm{MgO}_{2} \mathrm{Si}$ \\
\hline Formelmasse / $\mathrm{g} \cdot \mathrm{mol}^{-1}$ & 708.04 & 637.46 & 386.92 \\
\hline Kristallsystem & monoklin & monoklin & monoklin \\
\hline Raumgruppe & $P 2{ }_{1} / \mathrm{c}$ & $P 2{ }_{1} / \mathrm{c}$ & $P 2{ }_{1} / \mathrm{n}$ \\
\hline Gitterkonstanten / $\AA$ & $\begin{array}{l}a=10.7415(1) \\
b=19.1055(1) \\
c=23.0551(1) \\
\gamma=102.641(1)^{\circ}\end{array}$ & $\begin{array}{l}a=9.9986(1) \\
b=10.3648(1) \\
c=18.4893(1) \\
\gamma=100.044(1)^{\circ}\end{array}$ & $\begin{array}{l}a=13.3526(6) \\
b=13.9403(6) \\
c=13.9275(6) \\
\gamma=112.204(1)^{\circ}\end{array}$ \\
\hline Zellvolumen / $\AA^{3}$ & $4616.72(5)$ & $1886.74(3)$ & $2400.21(18)$ \\
\hline $\bar{Z}$ & 4 & 2 & 4 \\
\hline Dichte (berechnet) / $\mathrm{g} \cdot \mathrm{cm}^{-3}$ & 1.019 & 1.122 & 1.071 \\
\hline Absorptionskoeffizient / $\mathrm{mm}^{-1}$ & 0.232 & 0.097 & 0.136 \\
\hline$F(000)$ & 1552 & 688 & 848 \\
\hline Kristallgröße / $\mathrm{mm}^{3}$ & $0.50 \times 0.42 \times 0.40$ & $0.62 \times 0.46 \times 0.38$ & $0.50 \times 0.30 \times 0.28$ \\
\hline Messbereich & $1.40 \leq \theta \leq 25.00^{\circ}$ & $2.07 \leq \theta \leq 27.50^{\circ}$ & $1.80 \leq \theta \leq 25.00^{\circ}$ \\
\hline Indexbereich & $\begin{array}{l}-12 \leq \mathrm{h} \leq 12 \\
-19 \leq \mathrm{k} \leq 22 \\
-27 \leq 1 \leq 26\end{array}$ & $\begin{array}{l}-9 \leq \mathrm{h} \leq 12 \\
-13 \leq \mathrm{k} \leq 13 \\
-24 \leq 1 \leq 21\end{array}$ & $\begin{array}{l}-14 \leq \mathrm{h} \leq 15 \\
-16 \leq \mathrm{k} \leq 16 \\
-14 \leq 1 \leq 16\end{array}$ \\
\hline Gemessene Reflexe & 27326 & 13964 & 14910 \\
\hline Unabhängige Reflexe & $8080\left[\mathrm{R}_{\mathrm{int}}=0.1273\right]$ & $4322\left[\mathrm{R}_{\text {int }}=0.0518\right]$ & $4220\left[\mathrm{R}_{\mathrm{int}}=0.1026\right]$ \\
\hline$T_{\max } / T_{\min }$ & $0.9128 / 0.8926$ & $0.9642 / 0.9426$ & $0.9629 / 0.9351$ \\
\hline Daten / Restraints / Parameter & 8080 / 0 / 397 & 4322 / 16 / 267 & $4220 / 0$ / 242 \\
\hline GOF auf $\mathrm{F}^{2}$ & 0.979 & 1.042 & 1.011 \\
\hline Finaler R-Wert $[I>2 \sigma(\mathrm{I})]$ & $\begin{array}{l}R 1=0.0666 \\
w R 2=0.1366\end{array}$ & $\begin{array}{l}R 1=0.0559 \\
w R 2=0.1487\end{array}$ & $\begin{array}{l}R 1=0.0623 \\
w R 2=0.1405\end{array}$ \\
\hline R-Wert (alle Daten) & $\begin{array}{l}R 1=0.1454 \\
w R 2=0.1671\end{array}$ & $\begin{array}{l}R 1=0.0795 \\
w R 2=0.1638\end{array}$ & $\begin{array}{l}R 1=0.1234 \\
w R 2=0.1676\end{array}$ \\
\hline Max. / min. Restelektronendichte / e. $\AA^{-3}$ & $0.373 /-0.374$ & $0.367 /-0.354$ & $0.468 /-0.341$ \\
\hline
\end{tabular}

